\title{
Comparative navigation system for collaborative architectural design
}

\author{
S. $\mathrm{Oh}^{1} \& \mathrm{~W} . \mathrm{Yeo}^{2}$ \\ ${ }^{1}$ Universal City Group, National Institute of Information and \\ Communications Technology, Japan \\ ${ }^{2}$ Graduate School of Engineering, Osaka University, Japan
}

\begin{abstract}
We investigated the concepts, strategies, and functions of a 3D virtual design environment for collaborative, real-time architectural design using our 3D comparative navigation system and virtual reality technology. The development of the 'comparison' concept has enabled interactive design in real time in a 3D computer environment. Since participants must be able to easily understand the proposed design, systems that help them gain this understanding are required. While comparison is an effective way to gain such an understanding, comparing one proposed design to another using existing systems is difficult because the user must operate their viewpoints separately. We therefore created a prototype system that displays different contents simultaneously while controlling the viewpoints automatically to facilitate content comparison. This comparative navigation system facilitates the comparison of proposed designs by displaying related parts of the designs automatically. In this paper, we describe the concepts, strategies, and functions of a 3D virtual design environment for collaborative, real-time architectural design that is based on our 3D comparative navigation system and real-time simulation technology. We also evaluate the advantages and disadvantages of using this design environment for collaborative architectural design.

Keywords: collaborative design, comparison, interactive, real-time simulation, virtual reality, digital archive, experience.
\end{abstract}




\section{Introduction}

The use of 3-dimensional computer graphic (3DCG) models as an architectural design tool has have been increasing in recent years. Awareness of design has been increasing, and awareness is essential for efficient architectural rendering and agreement between participants. Both specialists and non-specialists can more effectively understand a design by using 3DCG.

The inspection of designs by participants and the demand for presentations are high. Various viewpoints need to be considered in collaborative design, not only the viewpoints of the enterprise body or designer. There is also an increasing demand for designers to be able to respond immediately to demands in presentations.

Collaboration up till now has involved the creation of still pictures or animations from a viewpoint assumed in advance. But it is difficult to guess all the assumptions for all the needs that the participants. Moreover, during collaboration responses cannot be immediately made to spontaneous needs.

Here we focus on a 3D real-time simulation engine as a design tool that solves these problems immediately. It would provide an effective, interactive, and rapid design platform. It could be applied to all stages of the design process and be used to check the designs at any time, anywhere, and at any stage. Its support of visualization and interactivity would enable good communication between the client and designer, thereby reducing misunderstandings. The realtime interactive previews it would enable should become a major part of the design process.

The rapid increase in the power of personal computers along with the drop in their prices has led to the migration of visual simulation and computer animation applications from expensive workstations to inexpensive PCs. We can now obtain faster rendering and higher quality results from PCs. The development of $3 \mathrm{D}$ real-time visual simulation has enabled the rendering of high-quality images at high speed.

Architectural design involves two major components, i.e., photorealistic and scenario scripting, which enable participants to feel a greater sense of realism. In the field of entertainment, movie makers and video-game programmers are investing a great deal of economic and human resources in developing a good interactive interface, i.e., a 3D real-time simulation engine. The entertainment field is expanding very quickly. This field has grown considerably over the past few years, and hardware and software are approaching perfection.

The aim of our research was to develop a good, interactive 3D development platform. It arose from an urgent need for 3D real-time simulation techniques that could be used to produce better architectural designs. We therefore focused on applying a real-time simulation engine to architectural design.

\subsection{Comparison for collaborative design}

The participants in a design project must understand the proposal on which they are working. Systems that help them gain this understanding are therefore 
required. Comparison is as an effective method for assisting understanding. For example, the representation of a person can be compared with that of an object to help the user better understand a certain object. By considering the differences, the user can recognize and understand an object. That is, the user compares and contrasts to clarify the areas with similarities and differences, enabling the user to recognize each similarity and difference. Therefore, we put considerable emphasis on comparison. A person can clarify the correlation, the effect, and the causal relation of objects by multilaterally comparing the objects. In addition, a person can deepen his or her understanding of each feature of the contents being compared, resulting in a better overall understanding of the whole.

Comparing one type of content to another using existing systems is difficult because users have to locate contents and observe them from separate viewpoints. With this in mind, we developed a prototype system that simultaneously displays various types of content while automatically controlling the viewpoints, enabling the user to easily compare them. Such a comparative navigation system facilitates comparison of design proposals by displaying their related parts automatically. Our 3D comparative navigation system is semantically a $3 \mathrm{D}$ extension of such a system. In the real world, people can walk through only one space, while in virtual space, a user can walk through many spaces at the same time. This is a key concept of our system, and it is intuitive and effective.

\subsection{Digital archives for comparison}

Early digital archives only digitized and stored text and photographs, while modern digital archives now include photographs, videos with text explanations, and 3DCG models. In the architectural field, for example, the use of 3D digital models for computer-aided-design/computer-aided-manufacturing has improved the efficiency of design, construction, and management. The 3D model can be of huge objects that one can walk around, such as places with historical architecture or archaeological sites. The digital archives described in this paper assume a 3D model, hence the term '3D digital archives.'

Many case studies reflecting the expansion of 3D digital archives have been presented at international conferences [1-4]. The main focus of these studies has been the construction of the archives. There have been few reports of research into how such archives can be used or how they can be experienced.

The main feature of 3D digital archives is that users do not simply see a flat 2D image of the contents out of context - they can see the contents from all angles in a natural setting. In addition, users can experience the contents as if they were in the real world. Moreover, they can run simulations and scientific investigations that would not be possible in the real world.

\section{Navigation function for comparison}

To gain an understanding by comparison, we need to consider the original purpose and objective first. The content to be used changes on the basis of the comparative purpose and comparative object. 
We have been examining various types of comparisons used in design projects over many years. The comparisons were made using various media, such as real-time simulation, animation, and still pictures. The functions required for comparison navigation were extracted by examining the comparison techniques used in the design projects. To generalize the comparison techniques and make them suitable for sharing, we arranged the main contents used for the comparisons on the basis of two viewpoints.

- Comparison of the differences in existence, form, and size to reveal the identity, similarities, heterogeneity, and features of 3D structures.

- Comparison of the changes in an object over time to reveal the similarities and differences at each stage, thus giving a picture of the change process.

The functions of comparison navigation were considered based on these two viewpoints. Users can compare different contents to help them understand particular content. By considering the differences, users can recognize and understand the content. They can also clarify the relationships, effects, and causal relations between different contents by comparing the contents multilaterally. In addition, the process of comparison makes it easier to identify different features of the content, encouraging greater understanding. The functions were arranged in accordance with the comparison purpose, the target content, the viewpoint setup, the viewpoint movement, the screen separation, and the content expression. Consequently, ten functions were identified for general comparison navigation; screen division comparison, photograph/model comparison, transparency change comparison, superposition comparison, model change comparison, camera viewing angle change, shadow display, comparison object insertion, measurement, and guide map display.

We developed a comparative navigation system that uses these functions and real-time simulation to facilitate interactive comparative studies of $3 \mathrm{D}$ architectural designs.

\section{Comparative navigation system}

In this section we describe the development of our comparative navigation system for $3 \mathrm{D}$ architectural design and our prototype system.

\subsection{Development}

We used various tools, including an authoring tool, a modeling tool, and an image-editing tool, to develop this system. The main technology used was realtime simulation technology based on virtual reality.

We developed an interface for a real-time simulation system and added it to the graphical user interface (GUI) using Virtools ${ }^{\circledR}$, which is a Microsoft $\mathrm{C}$ DirectX Graphics Application Programming Interface (API) used as an authoring tool in the game development environment. Producing architectural simulation is an impossible task for non-professional programmers, and architectural designers basically have no idea of how to produce a 3D scene. Virtools' building block system was specifically designed to meet the needs of cutting-edge interactive 
$3 \mathrm{D}$ development and is the only interactive $3 \mathrm{D}$ authoring tool accessible to nonprogrammers. Building Block is a subprogram packaged in a dynamic link library.

We assign building blocks in the scene as object behaviors through the visual authoring interface. We can then modify the scene by linking building blocks (using another subprogram). Specifically designed to meet the production needs of cutting-edge architectural simulation, the building block system provides the groundwork and tools users need to unleash their creativity and harness the full potential of the 3D real-time simulation engine. Users can import industrystandard media files to the building block system as 3D models, textures, characters, sets, and sounds. They can attach behaviors to these entities to create interactions. They can control and tweak the behaviors to form a higher-level element that forms the foundation for interactivity or simulation. The building block system's intuitive GUI enables real-time 3D environments to be designed and instantly experienced in an interactive sophisticated manner. Behaviors can be collected from a multitude of sources (libraries, other projects, etc.) and be exchanged over the Web. This system's open architecture makes all the behaviors compatible, so they can be recombined with existing modules. The interface is constructed using Virtools ${ }^{\circledR}$ scripts, as shown in Figure 1.
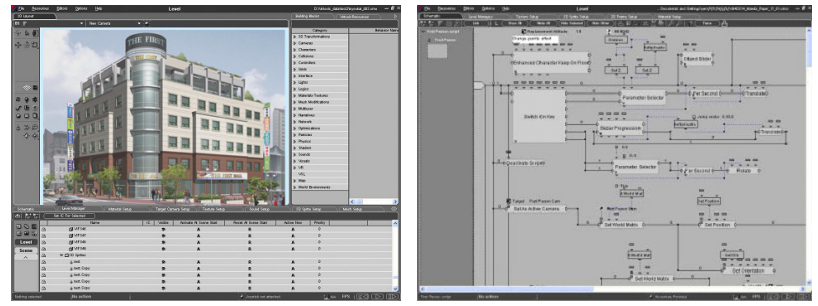

Figure 1: $\quad$ Scripts for real-time simulation interface using Virtools ${ }^{\circledR}$.

Specifically, we used a note PC with a 4.3-GHz CPU, an ATI Radeon 9800 GPU, and 2-GB RAM. To enable us to perform the rendering in real time with smooth movement, the rendering had to be done at no less than ten frames per second. This system was designed for comparative navigation in collaborative architectural design. The system had to meet three conditions in particular for it to support real-time simulation.

1. High-speed rendering: There is a trade-off between a high sense of reality and high-speed rendering as the system may not have sufficient performance for both. Priority was thus given to rendering at high speed to archive real-time simulation. The system also had to ensure the highest sense of reality.

2. Lightweight $3 D$ data: One way to increase the rendering speed is by reducing the weight of the data. A balance needs to be found between sufficient data speed and a sense of reality. Moreover, the user should not feel stress while using the system through the Internet. That is, the system should be able to read the 3D data in less than 90 seconds. This can be achieved by selecting suitable hardware and software. 
3. Easy operation interface: The interface should be easy to operate. In situations where operation does not catch up with rendering, the rendering speed should be reduced. Moreover, the interface should be immediately usable, even by a first-time user.

\subsection{System outline}

This system enables a general user to construct a building in virtual space. The internal and external design models for the building are first recorded on the modeling server. If the name of the building that the user wants to access and use for the server for network distribution is already defined, interior and exterior space models suitable for it are retrieved from the modeling server. Moreover, if a design proposal to use it is already defined, the model is loaded from the modeling server and sent to the user. The user can design the proposal while manipulating the model in virtual space. The user can also record the data on the server using his or her ID. The system concept is illustrated in Figure 2.

1. The user accesses the web and selects a model from a menu.

2. The web server sends a request to the database management system (DBMS) for information about the requested model.

3. The DBMS sends the uniform resource locator (URL) of the file server containing the requested information.

4. The web server sends a request to the file server for the target file.

5. The file server sends the requested file.

6. The web server displays the file contents to the user.

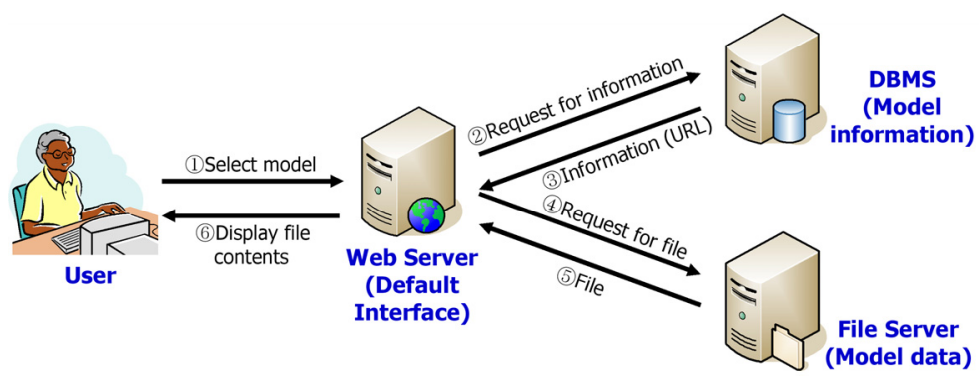

Figure 2: $\quad$ System concept.

\subsection{Prototype}

The prototype system includes the features of a traditional walk-through simulation and is especially aimed at enabling users to experience the archive information through browsing in the following ways.

- As the use freely walks through the archives, the system provides on-demand comparative views of related content.

- If the user 'collides' with a model wall, a collision detection function generates a rebounding effect, similar to the impression received when colliding with a wall in an actual building. 
- The user's view is fixed at eye level by a 'gravity' function. The user does not 'sink' into the floor or 'float' above it but rather walks around as in the real world.

- The user can compare the various types of archive contents interactively. As soon as the user selects contents to be compared, the system displays them.

- The user can select from several interfaces - a mouse, a keyboard, a game controller, and a space/mouse traveler.

The process of making a comparative navigation system for a $3 \mathrm{D}$ architectural structure experience can be divided into five steps (Figure 3).

1. Gather into a database all data created by the modeling; 3D models, GIS models, photos, etc.

2. Convert the data into models using modeler for Virtools ${ }^{\circledR}$. Group the models together to form scenes within a circumferential environment setting, a material setting, and alterable models.

3. Assign attributes and behaviors to the objects and scenes in the setting, including the camera settings, light settings, collision detection settings, level of detail (LOD) settings and gravity settings. Also create the GUI and system functions.

4. Test and debug the system using imported $3 \mathrm{D}$ objects.

5. Save in the database the behavior blocks, scenes, etc. constructed during testing.

The GUI operates as shown in Figure 4. We optimized the comparison navigation, which is the most important concept of this system, so that it can be fully utilized. For example, to enable many types of content to be compared, we made it easy to switch between one-screen, two-screen and four-screen mode comparison.

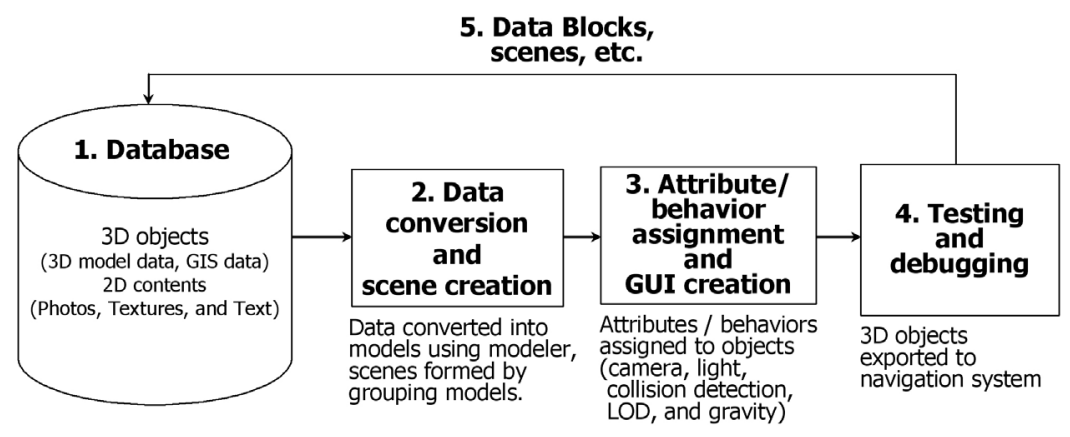

Figure 3: Steps in creating comparative navigation system.

\subsection{Functions}

The main functions of the system are 3D space move, plug-in, concurrent comparison navigation, and cross-section viewing. 


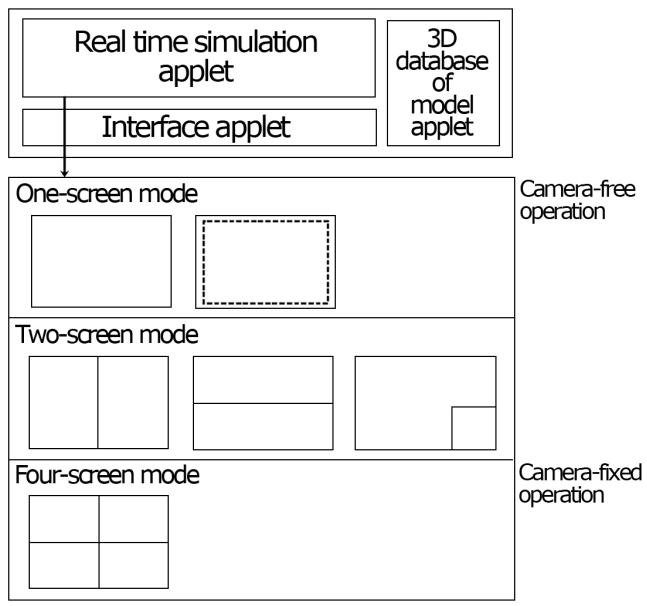

Figure 4: $\quad$ Operation of GUI.

\subsubsection{D space move}

The basic functions for moving in 3D space are 'free walk' and 'free flight'. They enable a user to examine a proposed design in 3D space. The user can freely start at any point and move freely in 3D space.

\subsubsection{Plug-in}

Using a system like that illustrated in Figure 2, a user can choose the data insertion function from a menu on the screen. The system then loads a 3D model that the user chose from the database for the design. The 3D model can be arranged freely. Moreover, the rearrangement is also possible after arrangement, rotation, and scale change.

\subsubsection{Concurrent comparison navigation}

Concurrent comparison navigation enables the user to comparatively examine a design proposal on one, two, or four screens by using easy key operations. In one-screen mode, the user can compare two proposed plans on the same screen. In two-screen mode, the user can compare two or more proposed designs by dividing the screen, as shown in Figure 4. In four-screen mode, the user can compare a proposed design with a photograph, etc.

\section{One-screen mode}

- Superposition comparison: The position and size of a structure can be compared by layering two types of content and changing the transparency of one of the layers.

- Comparison by inserting objects: Size and scale can easily be grasped by inserting and displaying a 3D object of a known size such as a piece of furniture or a person.

\section{Two-screen mode}

- Vertical/Horizontal screen division: Users can compare contents while walking in virtual space by displaying two type of contents on the same screen 
simultaneously. The user can then understand the composition of the space through two camera views. The display can be divided between left and right or between top and bottom. A controller that operates both cameras simultaneously is shown at the center of the display. As the user traverses the same route on both screens, each screen displays the content from the appropriate viewpoint.

- Guide screen mode: Users can display another proposed design on a small guide screen while focusing on the design of interest on the main screen. The designs can be switched between screens.

\section{Four-screen mode}

- Photograph/model comparison: Users can select a photograph from a photograph database, and the selected photograph is displayed in the lower left space. At the same time, models with the same viewpoint as the photograph can be displayed in the two upper spaces. Using this function enables the user to simultaneously compare the contents of various media, such as 3D models and $2 \mathrm{D}$ photographs. The user can also position the camera at a particular viewpoint.

\subsubsection{Cross-section view}

Users can display a cross-section view of a structure by controlling the cutting plane. Using this function enables the user to understand the inner structure in detail.

\section{Conclusion}

In conclusion, as the first step in developing a shared $3 \mathrm{D}$ environment that enables users to interact and understand architectural 3D models collaboratively, we have developed a prototype of an interactive navigation system that supports comparison. We have thus prepared the basic technology for experiencing architectural spaces by quickly examining designs through the Internet. Our comparative navigation system equipped with a $3 \mathrm{D}$ database makes it possible to use 3D data and comparison functions for various purposes in architectural design. Furthermore, the interactive interface built into the real-time rendering system enables a knowledge-exchange architectural design system to be developed, thus providing alternatives to traditional architectural design systems. By using this system, users can experience 3D contents comparison by quickly examining contents through the Internet.

We plan to expand the amount of content that can be compared by developing a parallel navigation system. We want to develop into a system that can perform various comparison for the purpose. For example, a user could not only compare one type of 3D content to another type of 3D content, but also compare 3D content with a photograph, with a texture, or with a video clip. We also plan to enable temporal comparison in addition to spatial comparison. We are considering enlarging the range of projects that can be analyzed, increasing the amount of data used to set up the model, and speeding up rendering during real-time simulation. A structure is need that displays only the data required to provide an adequate explanation to avoid having to handle too much data and having to provide an overly complicated interface. This should enable more 
effective presentations for specific purposes, depend on processing efficiency. We also plan to investigate how the experience of presence in a $3 \mathrm{D}$ virtual space can be achieved through easy operation of the interface.

\section{References}

[1] Beraldin, J.A., Picard, M., El-Hakim, S.F., Godin, G., Valzano, V., Bandiera, A. and Latouche, C., Virtualizing a Byzantine Crypt by Combining High-resolution Textures with Laser Scanner 3D Data, 8th International Conference on Virtual Systems and MultiMedia, pp. 3-14, 2002.

[2] Kalnins, R.D., Markosian, L., Meier, B.J., Kowalski, M.A., Lee, J.C., Davidson, P.L., Webb, M., Hughes, J.F. and Finkelstein, A., WYSIWYG NPR; Drawing Strokes Directly on 3D Models, ACM SIGGRAPH, pp. 755-762, 2002.

[3] Pollefeys, M., Van Gool, L., Vergauwen, M., Cornelis, K., Verbiest, F. and Tops, J., 3D Recording for Archaeological Fieldwork, IEEE Computer Graphics and Application, pp. 20-27, 2003.

[4] Schnabel, M.A., Kuan, S. and Li, W., 3D Scanning, Digital Modeling, Rapid Prototyping and Physical Depiction, 9th International Conference on Computer Aided Architectural Design Research in Asia, pp. 227-237, 2004.

[5] Chen, E.S., QuickTime VR - An Image-based Approach to Virtual Environment Navigation, ACM SIGGRAPH Computer Graphics, pp. 29-38, 1995.

[6] Sagawa, R., Nishino, K., Wheeler, M.D. and Ikeuchi, K., Parallel processing of range data merging, IEEE/RSJ International Conference on Intelligent Robots and System, Vol.1, 577-583, 2001.

[7] Knight, M.W. and Brown, A.G.P., Navrgate X, Naturalistic Navigation Metaphor for Large Scale Virtual Environments, 8th International Conference on Computer Aided Architectural Design Research in Asia, pp. 625-629, 2003.

[8] Yeo, W., Yuda, Y., Oh, S., Kaga, A., Sasada, T. and Sato, R., The Development of an Environmental Design Helping System Using Realtime Simulation guide, 9th International Conference on Computer Aided Architectural Design Research in Asia, pp. 947-955, 2004.

[9] Palmon, O., Oxman, R., Shahar, M. and Weiss P. L., Virtual environments in design and evaluation, Computer Aided Architectural Design Future2005, pp. 145-154, 2005.

[10] Oh, S., Tanaka, K. and Sasada, T., 3D Digital Archive Experience for Historical Architectures, International Conference on Education and Research in Computer Aided Architectural Design in Europe, pp. 573-580, 2005. 